Pacific Journal of Mathematics

ANALYTIC METHODS IN THE STUDY OF ZEROS OF 


\section{ANALYTIC METHODS IN THE STUDY OF ZEROS OF POLYNOMIALS}

\section{ZALMAN RUBinstein}

Several analytic methods are used to obtain estimates for part or all zeros of a polynomial with complex coefficients and for linear combinations of polynomials. Some results of Biernacki, Montel and Specht are strengthened or generalized. Some results about the location of zeros of linear combinations of polynomials are also obtained.

\section{Cauchy type estimates.}

THEOREM 1. Let $P(z)=z^{n}+a_{1} z^{n-1}+\cdots+a_{n}$ be a polynomial with complex coefficients. Let $\beta_{2}^{\prime} \geqq \beta_{3}^{\prime} \geqq \cdots \geqq \beta_{n}^{\prime}$ be the ordered positive numbers $\left|b_{i}\right|=\left|a_{i} \gamma^{-i}\right|, \gamma>0, i=2, \cdots, n$, then all the zeros of the polynomial $P(z)$ are in the union of the two circles:

$$
|z|<\gamma\left(1+\sigma_{1}\right) \text { and }\left|z+a_{1}\right| \leqq \gamma
$$

where

$$
\sigma_{1}=\beta_{2}^{\prime}-\frac{\delta_{2}^{\prime}}{1+\beta_{2}^{\prime}}-\frac{\delta_{3}^{\prime}}{\left(1+\beta_{2}^{\prime}\right)^{2}}-\cdots-\frac{\delta_{n}^{\prime}}{\left(1+\beta_{2}^{\prime}\right)^{n-1}}
$$

with

$$
\delta_{i}^{\prime}=\beta_{i}^{\prime}-\beta_{i+1}^{\prime}, \beta_{n+1}^{\prime}=0
$$

Proof. It is well known (See e.g. [4]), that all zeros of the polynomial $P(z)$ are in the union of the two circles $\left|z+a_{1}\right| \leqq \gamma$ and $|z| \leqq \gamma\left(1+\beta_{2}^{\prime}\right)$. Let $\zeta$ be a zero of the polynomial $P(z)$. We may assume that $|\zeta|=\gamma r$, where $1<r \leqq 1+\beta_{2}^{\prime}$. The inequality

$$
\left|\zeta^{n}+a_{1} \zeta^{n-1}\right| \leqq\left|a_{2}\right||\zeta|^{n-2}+\cdots+\left|a_{n}\right|
$$

yields

(1) $r^{n-1}\left|\zeta+a_{1}\right| \leqq \gamma\left(\left|b_{2}\right| r^{n-2}+\cdots+\left|b_{n}\right|\right) \leqq \gamma\left(\beta_{2}^{\prime} r^{n-2}+\cdots+\beta_{n}^{\prime}\right)$,

since $\beta_{i}^{\prime}, i=2, \cdots, n$, are decreasing and $r>1$. Multiplying both sides of the inequality $(1)$ by $(r-1) r^{-(n-1)}$ we get

$$
(r-1)\left|\zeta+a_{1}\right| \leqq \gamma\left(\beta_{2}^{\prime}-\frac{\delta_{2}^{\prime}}{r}-\cdots-\frac{\delta_{n}^{\prime}}{r^{n-1}}\right) .
$$

Received March 20, 1962. This research was supported by the National Science Foundation under Contract No. NSF-G 11592 with the University of Maryland. 
Since $r \leqq 1+\beta_{2}^{\prime}$ and $\delta_{i}^{\prime} \geqq 0$,

$$
(r-1)\left|\zeta+a_{1}\right| \leqq \gamma\left(\beta_{2}^{\prime}-\frac{\delta_{2}^{\prime}}{1+\beta_{2}^{\prime}}-\cdots-\frac{\delta_{n}^{\prime}}{\left(1+\beta_{2}^{\prime}\right)^{n-1}}\right) .
$$

Suppose $\left|\zeta+a_{1}\right|>\gamma$, then

$$
r<1+\beta_{2}^{\prime}-\frac{\delta_{2}^{\prime}}{1+\beta_{2}^{\prime}}-\cdots-\frac{\delta_{n}^{\prime}}{\left(1+\beta_{2}^{\prime}\right)^{n-1}}
$$

and

$$
|\zeta|=r \gamma<\gamma+\gamma\left(\beta_{2}^{\prime}-\frac{\delta_{2}^{\prime}}{1+\beta_{2}^{\prime}}-\cdots-\frac{\delta_{n}^{\prime}}{\left(1+\beta_{2}^{\prime}\right)^{n-1}}\right) .
$$

Consequently all the zeros of the polynomial $P(z)$ which are outside the circle $\left|\zeta+a_{1}\right| \leqq \gamma$ lie inside the circle (2).

One notes that this result can be repeatedly improved replacing $\beta_{2}^{\prime}$ by $\sigma_{1} \leqq \beta_{2}^{\prime}$ in the proof of Theorem 1 . The last result improves the known estimate used. As an immediate consequence from Theorem 1 we obtain that all the zeros of the polynomial $P(z)$ are in the region

$$
[|z|<\gamma(1+\sigma)] \cap\left(\left[\left|z+a_{1}\right| \leqq \gamma\right] \cup\left[|z|<\gamma\left(1+\sigma_{1}\right)\right]\right) .
$$

(See remark following the proof of theorem 2).

TheOREM 2. Let $P(z)=z^{n}+a_{1} z^{n-1}+\cdots+a_{n}$, be a polynomial with complex coefficients. Let $\gamma>0, b_{i}=a_{i} \gamma^{-i}, i=1, \cdots, n$. Assume furthermore that $\beta_{i}, i=1, \cdots, n, \beta_{1} \geqq \beta_{2} \geqq \cdots \geqq \beta_{n}$, are the ordered numbers $\left|b_{i}\right|$.

Define

$$
0 \leqq \alpha=\max _{2 \leqq i \leqq n} \frac{\beta_{i}}{\beta_{i-1}} \leqq 1,
$$

where the maximum is taken over all $i$ such that $\beta_{i-1} \neq 0$. Denote

$$
\sigma_{2}=\beta_{1}-\frac{\delta_{1}^{\prime \prime}}{1+\beta_{1}}-\frac{\delta_{2}^{\prime \prime}}{\left(1+\beta_{1}\right)^{2}}-\cdots-\frac{\delta_{n}^{\prime \prime}}{\left(1+\beta_{1}\right)^{n}}
$$

where

$$
\delta_{i}^{\prime \prime}=\alpha \beta_{i}-\beta_{i+1} \geqq 0, i=1, \cdots, n, \beta_{n+1}=0 ;
$$

then all the zeros of the polynomial $P(z)$ are in the circle

$$
|z| \leqq \max \left(\gamma\left(\alpha+\sigma_{2}\right), \gamma\right) \text {. }
$$

Proof. Let $\zeta$ be a zero of $P(z)$. We may assume that 


$$
\gamma\left(\alpha+\sigma_{2}\right) \leqq|\zeta|=\gamma r<\gamma\left(1+\beta_{1}\right), \quad r \geqq 1 .
$$

The equality $\zeta^{n}=-a_{1} \zeta^{n-1}-\cdots-a_{n}$ implies

$$
\gamma^{n} r^{n} \leqq\left|a_{1}\right| \gamma^{n-1} r^{n-1}+\cdots+\left|a_{n}\right|=\gamma^{n}\left(\left|b_{1}\right| r^{n-1}+\cdots+\left|b_{n}\right|\right) .
$$

Hence,

$$
r^{n} \leqq \beta_{1} r^{n-1}+\beta_{2} r^{n-2}+\cdots+\beta_{n} .
$$

Also since $r \geqq \alpha$, we have

$$
\begin{aligned}
r^{n}(r-\alpha) \leqq \beta_{1} r^{n}-r^{n-1}\left(\alpha \beta_{1}-\beta_{2}\right)-\cdots-r^{2}\left(\alpha \beta_{n-2}-\beta_{n-1}\right) \\
-r\left(\alpha \beta_{n-1}-\beta_{n}\right)-\alpha \beta_{n}=\beta_{1} r^{n}-\delta_{1}^{\prime \prime} r^{n-1}-\cdots-\delta_{n-1}^{\prime \prime}-\delta_{n}^{\prime \prime}
\end{aligned}
$$

Since $\alpha+\sigma_{2} \leqq r,(5)$ implies

$$
\frac{\delta_{1}^{\prime \prime}}{r}+\cdots+\frac{\delta_{n}^{\prime \prime}}{r^{n}} \leqq \beta_{1}-r+\alpha \leqq \beta_{1}-\sigma_{2} .
$$

Taking into account (3) and (6) we get

$$
\frac{\delta_{1}^{\prime \prime}}{r}+\cdots+\frac{\delta_{n}^{\prime \prime}}{r^{n}} \leqq \frac{\delta_{1}^{\prime \prime}}{1+\beta_{1}}+\cdots+\frac{\delta_{n}^{\prime \prime}}{\left(1+\beta_{1}\right)^{n}} .
$$

Since $\delta_{i}^{\prime \prime} \geqq 0$ it follows now that $r \geqq 1+\beta_{1}$ which contradicts the assumption made in (4). Hence $|\zeta| \leqq \gamma\left(1+\sigma_{2}\right)$. If however, $r<1$ then $|\zeta|<\gamma$. Theorem 2 strengthens a result due to Specht [6], $|z|<\gamma(1+\sigma)$, where

$$
\sigma=\beta_{1}\left(\frac{\beta_{1}}{1+\beta_{1}}+\frac{\beta_{2}}{\left(1+\beta_{1}\right)^{2}}+\cdots+\frac{\beta_{n}}{\left(1+\beta_{1}\right)^{n}}\right) .
$$

One verifies easily that $\alpha+\sigma_{2} \leqq 1+\sigma$.

2. Estimates of at least $p$ zeros $(1 \leqq p \leqq n)$ for a polynomial of degree $n$. It is known, [2] p. 110, that is the coefficients $a_{0}, a_{1}, \cdots$, $a_{p-1}, a_{p+h}$ are fixed, then $p$ zeros of the polynomial

$$
Q(z)=a_{n} z^{n}+\cdots+a_{0}
$$

are bounded. Various bounds for at least $p$ zeros of $Q(z)$, as functions of these coefficients, were obtained by different authors ([2] Chap. VIII).

Lemma 1. (Montel) [2] p. 111-112. Let the polynomial $Q(z)$, defined in (7), have zeros $z_{1}, \cdots, z_{n}$, such that $\left|z_{1}\right| \geqq\left|z_{2}\right| \geqq \cdots \geqq$ $\left|z_{n}\right|$. Let 


$$
Q_{p}(z)=\frac{Q(z)}{\left(z_{1}-z\right) \cdots\left(z_{n-p}-z\right)}=\sum_{j=0}^{p} a_{j}^{(p)} z^{j}
$$

Define $r_{p}=\left|z_{n-p+1}\right|$. Then

(8) $\left|a_{k}^{(p)}\right| \leqq r_{p}^{-(n-p)} \sum_{j=0}^{k} C(n-p+j-1, j)\left|a_{k-j}\right| r_{p}^{-j}, \quad k=0,1, \cdots, p$.

Theorem 3. Let $Q(z)$ be a complex polynomial of degree $n$ defined in (7), $a_{n} \neq 0,1 \leqq p \leqq n$. Let $r(p)$ be the greatest positive root of the equation

$$
(z-\rho)(z-1)^{n-p}-S\left(p, q^{\prime}, \rho\right)=0
$$

where $0<\rho \leqq 1$ and

$$
S\left(p, q^{\prime}, \rho\right)=\max _{0 \leqq k \leqq p-1}\left(\sum_{j=0}^{k}\left(\left|\frac{a_{j}}{a_{n}}\right|^{q^{\prime}}\right)^{1 / q^{\prime}} \rho^{-(p-k-1)}\right), q^{\prime} \geqq 1 .
$$

Then at least $p$ zeros of the polynomial $Q(z)$ are in the circle

$$
|z| \leqq r(p)
$$

Proof. We apply the Holder inequality to (8) and after some simple transformations we obtain

$$
\begin{aligned}
\left|a_{k}^{(p)}\right| \leqq & r_{p}^{-(n-p)}\left[\sum_{j=0}^{k}\left(C(n-p+j-1, j) r_{p}^{-j}\right)^{p^{\prime}}\right]^{1 / p^{\prime}}\left[\sum_{j=0}^{k}\left|a_{k-j}\right|^{q^{\prime}}\right]^{1 / q^{\prime}} \\
\leqq & r_{p}^{-(n-p)} \sum_{j=0}^{k} C(n-p+j-1, j) r_{p}^{-j}\left[\sum_{j=0}^{k}\left|\alpha_{k-j}\right|^{q^{\prime}}\right]^{1 / q^{\prime}} \\
& 1 / q^{\prime}+1 / q^{\prime}=1 .
\end{aligned}
$$

Since $r(p) \geqq 1$, without loss of generality we may assume that $r_{p}>1$. We replace the first sum in the right hand side of (11) by

$$
\sum_{j=0}^{\infty} C(n-p+j-1, j) r_{p}^{-j}=\left(1-\frac{1}{r_{p}}\right)^{-(n-p)} .
$$

By (11) and (12) we get

$$
\left|a_{k}^{(p)}\right| \leqq\left(r_{p}-1\right)^{-(n-p)}\left[\sum_{j=0}^{k}\left|a_{j}\right|^{q^{\prime}}\right]^{1 / q^{\prime}}
$$

It is known (See e.g. [4]) that all the zeros of $Q_{p}(z)$, and in particular $z_{n-p+1}$, satisfy the inequality

$$
r_{p}=\left|z_{n-p+1}\right| \leqq \max _{0 \leqq k \leqq p-1}\left(\left|\frac{a_{k}^{(p)}}{a_{p}^{(p)}}\right| \frac{x_{1}}{x_{p-k}}+\frac{x_{p-k+1}}{x_{p-k}}\right)
$$

for any $x_{i}>0, i=1, \cdots, p, x_{p+1}=0$. Taking into account that $a_{p}^{(p)}$ 
$=(-1)^{n-p} a_{n}$ and using (13) and (14), with $x_{i}=\rho^{i}, i=1, \cdots, p, 0<$ $\rho \leqq 1$, we obtain

$$
r_{p} \leqq \max _{0 \leqq k \leqq p-1}\left[\left(r_{p}-1\right)^{-(n-p)}\left(\sum_{j=0}^{k}\left|\frac{a_{j}}{a_{n}}\right|^{q^{\prime}}\right)^{1 / q^{\prime}} \rho^{-(p-k-1)}+\rho\right] .
$$

Using the definition of $S\left(p, q^{\prime}, \rho\right)$, the equivalent to (15) is

$$
\left(r_{p}-\rho\right)\left(r_{p}-1\right)^{n-p} \leqq S\left(p, q^{\prime}, \rho\right) .
$$

It follows now easily that $r_{p} \leqq r(p)$.

COROLlaRY 1. Theorem 3 includes, as particular cases, a result due to Marden ([2] p.113) for $\rho=1$, and a result due to Montel ([2] Th. 32,1) for $q^{\prime} \rightarrow \infty, \rho=1$.

Proceeding as in the proof of Theorem 3, using this time the estimate

$$
\left|\frac{a_{k}^{(p)}}{a_{p}^{(p)}}\right|<\frac{N_{p} r_{p}^{n-p}}{\left(1+N_{p}\right)\left(r_{p}-1\right)^{n-p}-N_{p} r_{p}^{n-p}}
$$

for all $k, r_{p}>1$ and

$$
N_{p}=\max _{0 \leqq j \leqq p-1}\left|\frac{a_{j}}{a_{p}}\right|,
$$

due to Montel (See [2] p. 115), one obtains:

Theorem 4. Let $Q(z)$ be the polynomial defined in (7). At least $p$ zeros of $Q(z)$ are in the circle $|z| \leqq r_{1}(p)$, where $r_{1}(p)$ is the positive root of the equation

$$
\left(1+\frac{1}{N_{p}}\right) \frac{z-\rho}{z+\rho^{1-p}-\rho}\left(1-\frac{1}{z}\right)^{n-p}-1=0 .
$$

For $\rho=1$, Theorem 4 gives an estimate due to Montel (See [2] p. 115). We remark that by a minimum argument it follows, that for $(p-1) N_{p} \leqq 1$ and $\rho=\left[(p-1) N_{p}\right]^{1 / p}$, Theorem 4 yields results better than those obtained by the classical formula.

Using estimates which involve a number of arbitrary parameters we obtain bounds for at least $p$ zeros for lacunary polynomials. We quote first a lemma.

Lemma 2. At least $p$ zeros, $1 \leqq p \leqq n$, of the polynomial $Q(z)$ $=a_{n} z^{n}+\cdots+a_{0}, a_{n} \neq 0$, lie in or on the circle $|z|=\rho^{\prime}$ where $\rho^{\prime}$ is the positive root of either of the two equations: 


$$
\left|a_{n}\right| z^{n}-\sum_{k=0}^{p-1} C(n-k-1, p-k-1)\left|a_{k}\right| z^{k}=0
$$

and

$$
\left|a_{p}\right| z^{p}-\sum_{k=0}^{p-1} C(n-k, p-k) \mid a_{k} z^{k}=0 .
$$

(16) is due to Montel, (17) is due to Van Vleck. A simultaneous proof of (16) and (17) was given by Markovitch [3]. We prove now the following:

TheOREM 5. Let $Q(z)=a_{n} z^{n}+\cdots+a_{0}, a_{p} a_{n} \neq 0,1 \leqq p \leqq n$. Let $r$ and $s$ be two numbers having the properties: $r \geqq n-p+1$ is the smallest number such that $a_{n-r} \neq 0 ; s, 1 \leqq s \leqq p$, is the smallest number such that $a_{p-s} \neq 0$. Then at least $p$ zeros of the polynomial $Q(z)$ lie in, either of the two circles:

$$
|z| \leqq \max \left[\rho^{r},\left(\sum_{j=r}^{n} C(j-1, p-n+j-1) \rho^{-(j-r)}\left|\frac{a_{n-j}}{a_{n}}\right|\right)^{1 / r}\right]
$$

and

$$
|z| \leqq \max \left[\rho^{s},\left(\sum_{j=s}^{p} C(n-p+j, j) \rho^{-(j-s)}\left|\frac{a_{p-j}}{a_{p}}\right|\right)^{1 / s}\right]
$$

for any $\rho>0$.

Proof. Denote $c_{k}=-C(k-1, p-n+k-1)\left|a_{n-k}\right| a_{n} \mid$. The left hand side of (16) can be written as

$$
z^{n}+c_{q} z^{n-q}+\cdots+c_{n}, q=n-p+1 .
$$

By our assumption, (20) is equivalent to

$$
z^{n}+c_{r} z^{n-r}+\cdots+c_{n}, c_{r} \neq 0 .
$$

It follows from the result proved in [5], with $x_{i}=\rho^{i}, i=1, \cdots, n$, $\rho>0$, that all the zeros of the polynomial (21) are in the union of the circle and the lemniscate defined by the inequalities

$$
|z| \leqq \rho^{r}
$$

and

$$
\left|z^{r}+c_{r}\right| \leqq \sum_{j=r+1}^{n} C(j-1, p-n+j-1)\left|\frac{a_{n-j}}{a_{n}}\right| \rho^{-(j-r)} .
$$

The inequality (18) follows applying (16) and the last result. To prove (19), we define $c_{p-k}=-C(n-k, p-k)\left|a_{k}\right| a_{p} \mid$ and proceed as 
before, using this time equality (17).

COROLlaRY 2. Let $Q(z)=a_{n} z^{n}+\cdots+a_{p} z^{p}+a_{0}, a_{0} a_{p} a_{n} \neq 0,1 \leqq p$ $\leqq n$. At least $p$ zeros of $Q(z)$ are in or on, either of the two circles:

$$
|z|=\left(C(n-1, p-1)\left|\frac{a_{0}}{a_{n}}\right|\right)^{1 / n}
$$

and

$$
|z|=\left(C(n, p)\left|\frac{a_{0}}{a_{p}}\right|\right)^{1 / p}
$$

These results are obtained from Theorem 5, with $r=n$ and $r=p$ respectively. Both results are due to Van Vleck. (See [2]).

\section{Linear combinations of polynomials.}

LEMMA 3. Let the polynomials $R(z)=z^{n}+\cdots+a_{n}$ and $S(z)=$ $z^{k}+\cdots+b_{k}, a_{n} b_{k} \neq 0$, have zeros $z_{i}, i=1, \cdots, n$, and $\zeta_{j}, j=1, \cdots$, $k$ respectively.

Let $F(z ; \lambda)=R(z)+\lambda S(z)$ have zeros $\eta_{1}(\lambda), \cdots, \eta_{l}(\lambda),\left|\eta_{1}\right| \leqq\left|\eta_{2}\right|$ $\leqq \cdots \leqq\left|\eta_{l}\right|$. If the circle $|z| \leqq \tau$ contains all the zeros of the polynomials $R(z)$ and $S(z)$ and $m$ zeros, $0 \leqq m<l$, of $F(z ; \lambda)$, then

$$
\prod_{i=m+1}\left|\eta_{i}(\lambda)\right| \leqq \frac{1}{C(\lambda)} \tau^{[(1+(|\lambda| / \mu)) n-(|\lambda| / \mu) k-m]},
$$

where $C(\lambda)$ equals $|1+\lambda|,|\lambda|$, or 1 according to whether $k=n$, $\mathrm{k}>n$, or $k<n$ respectively, provided

$$
\mu=\min _{0 \leqq \theta \leqq 2 \pi}\left|\frac{R\left(\tau e^{i \theta}\right)}{S\left(\tau e^{i \theta}\right)}\right|-1>0 .
$$

Proof. Applying Jensen's formula to the polynomials $R(z), S(z)$ and $F(z ; \lambda)$ we obtain (Omitting the parameter $\lambda$ in the notation for $F)$ :

$$
\begin{gathered}
n \log \tau=\frac{1}{2 \pi} \int_{0}^{2 \pi} \log \left|R\left(\tau e^{i \theta}\right)\right| d \theta \\
k \log \tau=\frac{1}{2 \pi} \int_{0}^{2 \pi} \log \left|S\left(\tau e^{i \theta}\right)\right| d \theta \\
\log \left(|C(\lambda)| \prod_{i=m+1}^{l}\left|\eta_{i}(\lambda)\right|\right)+m \log \tau=\frac{1}{2 \pi} \int_{0}^{2 \pi} \log \left|F\left(\tau e^{i \theta}\right)\right| d \theta .
\end{gathered}
$$


Using the formulas (23) and the inequality $\log \left(\alpha x_{1}+\beta x_{2}\right) \geqq \alpha \log x_{1}$ $+\beta \log x_{2}$ with $x_{1}=|R|+|\lambda S|, x_{2}=|S|$ and

$$
\alpha=\frac{|R|-|S|}{|R|-|S|+|\lambda S|}, \alpha+\beta=1,
$$

after a few transformations we deduce the inequalities

$$
\begin{aligned}
& \int_{0}^{2 \pi} \log |F(z)| d \theta \leqq \int_{0}^{2 \pi} \log (|R(z)|+|\lambda S(z)|) d \theta \\
& \quad \leqq \int_{0}^{2 \pi} \frac{\log \left|R\left(\tau e^{i \theta}\right)\right|-\log \left|S\left(\tau e^{i \theta}\right)\right|}{\alpha(\theta, \lambda)} d \theta+\int_{0}^{2 \pi} \log \left|S\left(\tau e^{i \theta}\right)\right| d \theta .
\end{aligned}
$$

On the other hand, by the assumption of the theorem it follows that

$$
\frac{1}{\alpha(\theta, \lambda)} \leqq 1+\frac{|\lambda|}{\mu}
$$

Hence

$$
\begin{aligned}
\int_{0}^{2 \pi} \log |F(z)| d \theta \leqq\left(1+\frac{|\lambda|}{\mu}\right) \int_{0}^{2 \pi} \log \left|R\left(\tau e^{i \theta}\right)\right| d \theta-\frac{|\lambda|}{\mu} \int_{0}^{2 \pi} & \log \left|S\left(\tau e^{i o}\right)\right| d \theta .
\end{aligned}
$$

Dividing (24) by $2 \pi$ and substituting the values from (23), we deduce the inequality

$$
\log \left(|C(\lambda)| \prod_{i=m+1}^{l}\left|\eta_{i}(\lambda)\right|\right)+m \log \tau \leqq\left(1+\frac{|\lambda|}{\mu}\right) n \log \tau-\frac{|\lambda|}{\mu} k \log \tau .
$$

The desired follows now after simple transformations. We remark that in case $\mu<0$, Lemma 3 is true interchanging $R$ and $S, \lambda$ and $1 / \lambda$. As a consequence of the lemma we have:

THEOREM 6. Under the assumptions of Lemma 3 all the zeros of the polynomial $F(z ; \lambda)$ are in the disc

$$
|z| \leqq \frac{1}{|C(\lambda)|} \tau^{[(1+|\lambda| / \mu) n-(|\lambda| / \mu) k-l+1]} .
$$

Proof.

$$
\begin{aligned}
& \max _{m+1 \leqq i \leqq l}\left|\eta_{i}(\lambda)\right|=\prod_{i=m+1}^{l}\left|\eta_{i}(\lambda)\right|\left(\prod_{i \neq i \max }\left|\eta_{i}(\lambda)\right|\right)^{-1} \\
& \leqq \frac{1}{|C(\lambda)|} \tau^{[(1+|\lambda| / \mu) n-(|\lambda| / \mu) k-l+1]}
\end{aligned}
$$

since $\left|\eta_{i}(\lambda)\right| \geqq \tau$ for $m+1 \leqq i \leqq l$. Some estimates for the zeros of linear combinations of polynomials can be derived by a con- 
tinuity argument. We denote by $D(k, n-k)$ the open domain in the extended complex $\lambda$-plane, for which the polynomial $F(z ; \lambda)=$ $R(z)+\lambda S(z)$ has exactly $k$ zeros with negative real part $(n-k$ zeros with positive real part), where $R(z)$ and $S(z)$ are fixed and $\operatorname{deg}(R+\lambda S)$ $=n, k=0,1, \cdots, n$. Some of the domains $D(k, n-k)$ may be empty. We quote two results to be used later.

Lemma 4. (Marden [2] p.54). The zeros of the linear combination

$$
f(z)=\lambda_{1} f_{1}(z)+\cdots+\lambda_{p} f_{p}(z)
$$

where $\lambda_{j} \neq 0, j=1,2, \cdots, p$, lie in the locus $\Gamma$ of the roots of the equation

$$
\lambda_{1}\left(z-\alpha_{1}\right)^{n_{1}}+\cdots+\lambda_{p}\left(z-\alpha_{p}\right)^{n_{p}}=0
$$

when the $\alpha_{1}, \cdots, \alpha_{p}$ vary independently over the circular regions $C_{1}, \cdots, C_{p}$ and where $C_{j}$ contains all the zeros of the polynomial $f_{j}(z)$. The following result is due to Walsh (see [2] p. 55).

Lemma 5. If the points $\alpha_{1}, \cdots, \alpha_{p}$ vary independently over the closed interiors of the circles $C_{1}, \cdots, C_{p}$ respectively, then the locus of the point.

$$
\alpha=\sum_{j=1}^{p} m_{j} \alpha_{j}
$$

where the $m_{j}$ are arbitrary complex numbers, will be the closed interior of a circle $C$ of center $c$ and radius $r$, where

$$
c=\sum_{j=1}^{p} m_{j} c_{j}, r=\sum_{j=1}^{p}\left|m_{j}\right| r_{j}
$$

and $c_{j}$ and $r_{j}$ denote respectively the center and radius of the circle $C_{j}$. We prove a preliminary result.

LEMMA 6. Let

$$
f(z, \lambda)=(z+a)^{n}+\lambda z^{p}, \mid \text { Re } a \mid \geqq 1, \text { arg } a=\alpha, p \leqq n .
$$

Then for all $\lambda,|\lambda| \leqq\left|\cos ^{p} \alpha\right|$, the polynomial $f(z, \lambda)$ has all its zeros in the same right, or left, half plane as the polynomial $(z+a)^{n}$.

Proof. By the remarks made following the proof of Theorem 6 it is sufficient to prove that the domain $D(k, n-k), k=0$ or $n$, which contains the origin, contains also the circle about the origin with radius $\left|\cos ^{p} \alpha\right|$. Substituting $z=i y$ in (25) and solving for $\lambda$ 
in the equation $f(i y, \lambda)=0$ we get

$$
|\lambda|=|i y+a|^{n-p}\left|\frac{i y+a}{y}\right|^{p} \geqq|\operatorname{Re} a|^{n-p}\left|i+\frac{a}{y}\right|^{p} .
$$

Since by elementary geometric considerations $|i+a| y|\geqq| \operatorname{Re} a|| a \mid$, we get $|\lambda| \geqq\left.|\operatorname{Re} a|^{n}|| a\right|^{p}$, hence the region $D$ which contains the origin contains also the circle $|\lambda|<\cos ^{p} \alpha \|\left.\operatorname{Re} a\right|^{n-p}$. Taking into account the conditions (25) the desired result follows.

THEOREM 7. Let $R(z)=z^{n}+\cdots+a_{n}, S(z)=z^{k}+\cdots+b_{k}, k \leqq$ $n$. Let the zeros of $R(z)$ and $S(z)$ lie in the discs $C_{i}:\left|z-c_{i}\right| \leqq r_{i}$, $i=1,2$ respectively, such that one of the following conditions holds:

$$
\begin{aligned}
& \text { (a) } \operatorname{Re}\left[\left(c_{2}-c_{1}\right)\right]-\left(r_{1}+r_{2}\right) \geqq 1 \\
& \text { (b) } \operatorname{Re}\left[\left(c_{2}-c_{1}\right)\right]+r_{1}+r_{2} \leqq-1 .
\end{aligned}
$$

Denote the circle $\left|z-\left(c_{2}-c_{1}\right)\right| \leqq r_{1}+r_{2}$ by $C_{3}$ and let $\min _{z \in \sigma_{3}} \mid \cos$ $\arg z \mid=A$. Then in case (a) all the zeros of the polynomial $R(z)+$ $\lambda S(z)$ for $|\lambda| \leqq A^{k}$, are in the region $R e z \leqq r_{2}+$ Re $c_{2}$, and in case (b) -in the region $R e z \geqq \operatorname{Re} c_{2}-r_{2}$.

Proof. By Lemma 4 all the zeros of the polynomial $R(z)+\lambda S(z)$ are in the locus of the zeros of the polynomial $g(z)=\left(z-\alpha_{1}\right)^{n}+$ $\lambda\left(z-\alpha_{2}\right)^{k}$, where $\alpha_{i}, i=1,2$ vary independently in $C_{i}$. By Lemma 5, $\alpha_{2}-\alpha_{1} \in C_{3}$ for any $\alpha_{i} \in C_{i}$. We may apply therefore Lemma 6 to the polynomial $g\left(\zeta+\alpha_{2}\right)=\left[\zeta+\left(\alpha_{2}-\alpha_{1}\right)\right]^{n}+\lambda \xi^{k}$. It results that in case (a) all the zeros of the polynomial $g\left(\zeta+\alpha_{2}\right)$ are in the region $\operatorname{Re} \zeta \leqq 0$ for $|\lambda| \leqq A^{k}$, and $\operatorname{Re} z=\operatorname{Re} \zeta+\operatorname{Re} \alpha_{2} \leqq \operatorname{Re} \alpha_{2} \leqq r_{2}+\operatorname{Re} c_{2}$.

Similarly, in case (b), the zeros of $g\left(\zeta+\alpha_{2}\right)$ are in the region $R e \zeta \geqq 0$. It is clear that if $k>n$, similar results can be obtained replacing $\alpha_{2}$ by $\alpha_{1}, \lambda$ by $1 / \lambda$ and $R$ by $S$.

Combining the last result with a similar result for the imaginary part of the zeros of $R(z)+\lambda S(z)$ we obtain:

COROLLARY 3. With the notations and assumptions of Theorem 7 suppose that one of the following holds

$$
\begin{aligned}
& \text { (a') } \quad \operatorname{Re}\left[\left(c_{2}-c_{1}\right)\right]-\left(r_{1}+r_{2}\right) \geqq 1 \\
& \left(\mathrm{~b}^{\prime}\right) \quad \operatorname{Im}\left[\left(c_{2}-c_{1}\right)\right]-\left(r_{1}+r_{2}\right) \geqq 1 .
\end{aligned}
$$

Denote

$$
A_{1}=\min \left(\min _{z \in O_{3}}|\cos \arg z|, \min _{z \in O_{3}}|\sin \arg z|\right),
$$


Then for $|\lambda| \leqq A_{1}^{k}$, in case $\left(\mathrm{a}^{\prime}\right)$ all the zeros of the polynomial $R(z)+$ $\lambda S(z)$ are in the quadrant Re $z \leqq r_{2}+\operatorname{Re} c_{2}, \operatorname{Im} z \leqq r_{2}+\operatorname{Im} c_{2}$, and in case $\left(\mathrm{b}^{\prime}\right)$ all the zeros of the above polynomial are in the quadrant $\operatorname{Re} z \geqq \operatorname{Re} c_{2}-r_{2}$, Im $z \geqq \operatorname{Im} c_{2}-r_{2}$.

The estimates based on the continuity argument can be further developed and it is possible to obtain bounded regions for the zeros under suitable restrictions upon the parameter $\lambda$.

We prove finally a result concerning the location of at least $p$ zeros of linear combinations of polynomials.

THEOREM 8. Let $R(z)$ and $S(z)$ be two polynomials of degree $n$ and $k$ with zeros $z_{i}$ and $\zeta_{j}$ respectively, $i=1, \cdots, n, j=1 \cdots, k$. Let the numbers $a, r, r_{1}, r_{2}, r_{3}, n_{1}, n_{2}, k_{1}, k_{2} ; n_{1}+n_{2}=n, k_{1}+k_{2}=k$ satisfy the following conditions:

(a) The polynomial $R(z)$ has $n_{1}$ zeros in the disc $C_{1}:|z-a| \leqq r_{1}$ $<r$ and $n_{2}$ zeros outside the disc $C_{1}$ which are in the disc $\mid z[\leqq r$.

(b) The polynomial $S(z)$ has $k_{1}$ zeros in the disc $C_{2} ;|z-a| \leqq r_{2}$ $<r$ which are also in the disc $|z| \leqq r$ and $k_{2}$ zeros outside the disc $C_{2}$ which are also outside the disc $|z-a| \geqq r_{3}>r$.

Suppose furthermore that one of the following conditions is satisfied:

(c) $r<a_{0}$ and $n_{1} \frac{r+a_{0}}{r-r_{1}}+n_{2} \frac{r-a_{0}}{2 r+a_{0}} \leqq k_{1} \frac{r-a_{0}}{r}+k_{2} \frac{r+a_{0}}{r-r_{3}}$

(c) $r>a_{0}$ and $n_{1} \frac{r+a_{0}}{r-r_{1}}+n_{2} \frac{r-a_{0}}{2 r} \leqq k_{1} \frac{r-a_{0}}{r+r_{2}}+k_{2} \frac{r+a_{0}}{r-r_{3}}$,

then the polynomial $F(z ; \lambda)=R(z)+\lambda S(z)$ has at least $n_{1}$ zeros in the disc $|z-a| \leqq r$, for any complex number $\lambda$.

Proof. A straightforward calculation yields the following results: (1) Let $z=a+r e^{i \theta}$, then

$$
\begin{aligned}
& M=\max _{0 \leqq \theta \leqq 2 \pi} \operatorname{Re}\left(\frac{z}{z-u}\right)=\frac{r^{2}-\left|a^{2}\right|+R e(a \bar{u}) \pm r|u|}{r^{2}-|u-a|^{2}} \\
& m=\min _{0 \leqq \theta \leqq 2 \pi} \operatorname{Re}\left(\frac{z}{z-u}\right)=\frac{r^{2}-|a|^{2}+\operatorname{Re}(a \bar{u}) \mp r|u|}{r^{2}-|u-a|^{2}}
\end{aligned}
$$

according to $|u-a| \lessgtr r$ respectively.

(2) Let $a=a_{0} e^{i \varphi}, z-a=r e^{i \theta}, u-a=\rho e^{i \varphi}$, then 


$$
\begin{aligned}
& M_{1}=\max _{0 \leqq \theta, \varphi \leqq 2 \pi} \operatorname{Re}\left(\frac{z}{z-u}\right)=\left\{\begin{array}{l}
\frac{r+a_{0}}{r-\rho} \text { for } \rho<r \\
\frac{r+a_{0}}{r-\rho} \text { for } \rho>r,|u|<r \\
\frac{r^{2}-a_{0} \rho-r\left|a_{0}-\rho\right|}{r^{2}-\rho^{2}} \text { for } \rho>r,|u|>r
\end{array}\right. \\
& \mathrm{m}_{1}=\min _{0 \leqq \theta, \varphi \leqq 2 \pi} \operatorname{Re}\left(\frac{z}{z-u}\right)=\left\{\begin{array}{l}
\frac{r-a_{0}}{r+\rho} \text { for } r>\rho,|u|<r \\
\frac{r^{2}-a_{0} \rho-r\left|a_{0}-\rho\right|}{r^{2}-\rho^{2}} \text { for } r>\rho,|u|>r \\
\frac{r+a_{0}}{r-\rho} \text { for } r<\rho .
\end{array}\right.
\end{aligned}
$$

(4) Let the polynomial $R(z)$ satisfy condition (a) of Theorem 8, then

$$
\begin{aligned}
& M_{2}=\max _{|z-a|=r} \frac{d}{d \theta} \arg R(z) \leqq n_{1} \frac{r+a_{0}}{r-r_{1}}+n_{2} \frac{r-a_{0}}{2 r+a_{0}} \text { for } r<a_{0} \\
& M_{2} \leqq n_{1} \frac{r+a_{0}}{r-r_{1}}+n_{2} \frac{r-a_{0}}{2 r} \text { for } r>a_{0} .
\end{aligned}
$$

(5) Let the polynomial $S(z)$ satisfy condition (b) of Theorem 8, then

$$
\begin{aligned}
& m_{2}=\min _{|z-a|=r} \frac{d}{d \theta} \arg S(z) \geqq k_{1} \frac{r-a_{0}}{r}+k_{2} \frac{r+a_{0}}{r-r_{3}} \text { for } r<a_{0} \\
& m_{2} \geqq k_{1} \frac{r-a_{0}}{r+r_{2}}+k_{2} \frac{r+a_{0}}{r-r_{2}} \text { for } r>a_{0} .
\end{aligned}
$$

By the results (4) and (5), the condition $\left(c_{1}\right)$ or $\left(c_{2}\right)$ implies that

$$
\max _{|z-a|=r} \frac{d}{d \theta} \arg \frac{R(z)}{S(z)} \leqq \max _{|z-a|=r} \frac{d}{d \theta} \arg R(z)-\min _{|z-a|=r} \frac{d}{d \theta} \arg S(z) \leqq 0 .
$$

Hence the $\arg R(z) / S(z)$, as $|z-a|=r$ and $z$ makes one turn in the positive direction, decreases monotonically.

$$
\underset{|z-a|=r}{\arg } \frac{R(z)}{S(z)}=2 \pi\left(n_{1}-k_{1}\right) .
$$

It follows now that

$$
\underset{|z-a|=r}{\Delta}\left(\arg \frac{R(z)}{S(z)}+\lambda\right) \geqq 2\left(n_{1}-k_{1}\right)
$$

for any complex number $\lambda$. Hence

$$
\underset{|z-a|=r}{\Delta} \arg (R(z)+\lambda S(z)) \geqq 2 \pi\left(n_{1}-k_{1}\right)+2 \pi k_{1}=2 \pi n_{1} .
$$


This completes the proof.

In the particular case $a=0, n_{2}=k_{2}=0$, at least $n$ zeros of the polynomial $F(z ; \lambda)$ are in the disc $|z| \leqq \max \left(\left(n r_{2}+k r_{1}\right) /(k-n), r_{2}\right)$ for $k>n$. The last result is due to Biernacki (See. [1]). If $a=0$ and the zeros of $R(z)$ and $S(z)$ are in the discs $\left|z-c_{i}\right| \leqq d_{i}$, it results from Theorem 8 , with $r_{i}=\left|c_{i}\right|+d_{i}, i=1,2$, that in the case $k>n$, at least $n$ zeros of the polynomial $F(z ; \lambda)$ are in the disc

$$
|z| \leqq \max \left(\frac{n d_{2}+k d_{1}}{k-n}+\frac{n\left|c_{2}\right|+k\left|c_{1}\right|}{k-n}, d_{2}+\left|c_{2}\right|\right) .
$$

This result is due to Jankowski [1].

\section{REFERENCES}

1. W. Jankowski Sur les zeros d'un polynome contenant un parametre arbitraire, Ann. Polon. Math., 3 (1957), 304-311.

2. M. Marden, The geometry of the zeros of a polynomial in a complex variable, Math. Surveys Number III, 1949.

3. D. Markovitch, Sur quelques limites superieures des modules des zeros d'un polynome, Math. Cluj XV (1939), 8-11.

4. M. Parodi, La localisation des valeurs caracteristiques des matrices et ses application, Paris Gauthier-Villars, 1959.

5. Z. Rubinstein The location of zeros of lacunary polynomials, Amer. Math. Soc. “Notices", 8 (1961), p. 369.

6. W. Specht, Die Lage der Nullstellen eines Polynoms II. Mat. Nachr. 16 (1957), 257-263. 



\section{PACIFIC JOURNAL OF MATHEMATICS}

\section{EDITORS}

\section{RalPh S. Phillips}

Stanford University

Stanford, California

M. G. Arsove

University of Washington

Seattle 5, Washington
J. Dugundji

University of Southern California Los Angeles 7, California

Lowell J. Paige

University of California

Los Angeles 24, California

\section{ASSOCIATE EDITORS}
E. F. BECKENBACH
D. DERRY
H. L. ROYDEN
E. G. STRAUS
T. M. CHERRY
M. OHTSUKA
E. SPANIER
F. WOLF

\section{SUPPORTING INSTITUTIONS}

\author{
UNIVERSITY OF BRITISH COLUMBIA \\ CALIFORNIA INSTITUTE OF TECHNOLOGY \\ UNIVERSITY OF CALIFORNIA \\ MONTANA STATE UNIVERSITY \\ UNIVERSITY OF NEVADA \\ NEW MEXICO STATE UNIVERSITY \\ OREGON STATE UNIVERSITY \\ UNIVERSITY OF OREGON \\ OSAKA UNIVERSITY \\ UNIVERSITY OF SOUTHERN CALIFORNIA
}

STANFORD UNIVERSITY

UNIVERSITY OF TOKYO

UNIVERSITY OF UTAH

WASHINGTON STATE UNIVERSITY

UNIVERSITY OF WASHINGTON

AMERICAN MATHEMATICAL SOCIETY

CALIFORNIA RESEARCH CORPORATION SPACE TECHNOLOGY LABORATORIES

NAVAL ORDNANCE TEST STATION

Printed in Japan by International Academic Printing Co., Ltd., Tokyo Japan 


\section{Pacific Journal of Mathematics}

\section{Vol. 13, No. 1 \\ March, 1963}

Frantz Woodrow Ashley, Jr., A cone of super-(L) functions............. 1

Earl Robert Berkson, Some metrics on the subspaces of a Banach space....

Felix Earl Browder and Walter Strauss, Scattering for non-linear wave

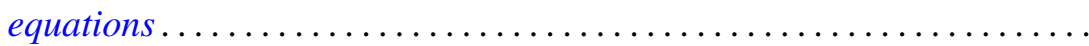

Edmond Darrell Cashwell and C. J. Everett, Formal power series ..........

Frank Sydney Cater, Continuous linear functionals on certain topological

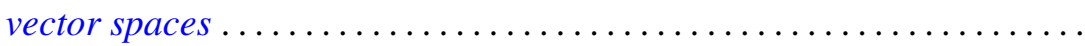

John Douglas Dixon, General group extensions ....................

Robert Pertsch Gilbert, On harmonic functions of four variables with

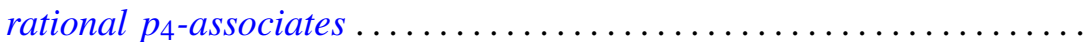

Irving Leonard Glicksberg, On convex hulls of translates ..............

Simon Hellerstein, On a class of meromorphic functions with deficient zeros

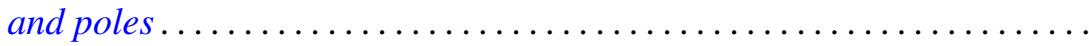

Donald William Kahn, Secondary cohomology operations which extend the

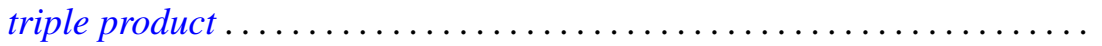

G. K. Leaf, A spectral theory for a class of linear operators .............

R. Sherman Lehman, Algebraic properties of the composition of solutions of partial differential equations ........................... 157

Joseph Lehner, On the generation of discontinuous groups ............. 169

S. P. Lloyd, On certain projections in spaces of continuous functions ...... 171 Fumi-Yuki Maeda, Generalized spectral operators on locally convex spaces ..................................

Donald Vern Meyer, $E^{3}$ modulo a 3-cell

William H. Mills, An application of linear programming to permutation groups.

Richard Scott Pierce, Centers of purity in abelian groups

Christian Pommerenke, On meromorphic starlike functions ...

Zalman Rubinstein, Analytic methods in the study of zeros of

polynomials...

B. N. Sahney, On the Nörlund summability of Fourier series

Tôru Saitô, Regular elements in an ordered semigroup . .

Lee Meyers Sonneborn, Level sets on spheres...........

Charles Andrew Swanson, Asymptotic estimates for limit point

problems .

Lucien Waelbroeck, On the analytic spectrum of Arens . .

Alvin (Murray) White, Singularities of a harmonic function of three

variables given by its series development .............

Kōichi Yamamoto, Decomposition fields of difference sets ...

Chung-Tao Yang, On the action of $\mathrm{SO}(3)$ on a cohomology manifold... 